\title{
Las ofrendas de concha de moluscos de la Pirámide de las Serpientes Emplumadas, Xochicalco, Morelos
}

\author{
The shell offerings inside the Pyramid of the Plumed Serpents in Xochicalco, Morelos
}

Emiliano Ricardo Melgar-Tísoc

Museo del Templo Mayor, Seminario 8, Centro, 06060 México, D.F.

Correspondencia: melgare@hotmail.com

Resumen. El propósito del presente trabajo fue evaluar los análisis llevados a cabo en los materiales de concha procedentes de la Pirámide de las Serpientes Emplumadas de Xochicalco, Morelos. Se identificaron 12 especies, 9 del Pacífico y 3 del Caribe. La tipología y las huellas de manufactura analizadas con microscopio electrónico de barrido (MEB) mostraron 2 patrones: una heterogeneidad y dispersión de las áreas de producción en la primera etapa, frente a la estandarización y concentración de las áreas de producción, quizás un taller bajo control de la élite, de la última etapa.

Palabras clave: tipología, manufactura, élite, bienes de prestigio.

\begin{abstract}
The aim of this study was to analyze the shell assemblage of the Pyramid of the Plumed Serpents in Xochicalco, Morelos. Twelve species were identified, 9 from the Panamic-Pacific province and the remaining 3 from the Caribbean Sea. Using Scanning Electron Microscopy (SEM), the typology and traces of working showed two patterns of manufacture: 1) variability and wide dispersal from the areas of production and, contrasting with this, 2) standardization of manufacture and centralization in those areas where, perhaps in latter stages, the shell workshop was under the control of elite members of the community.
\end{abstract}

Key words: typology, manufacture, elite, prestige goods.

\section{Introducción}

El presente trabajo aborda los diversos análisis de los objetos de concha de Xochicalco, Morelos, pero enfocándolos solamente en los que proceden de las ofrendas halladas en la Pirámide de las Serpientes Emplumadas (Fig. 1A), cuyos relieves hacen de esta estructura la más conocida de este asentamiento amurallado del Epiclásico -periodo que va del 600 al 900 d.C. (González et al., en prensa). Dicho edificio está ubicado en la parte este de la Plaza Principal, mientras su acceso abre al oeste mirando hacia la Acrópolis. Cabe señalar que se eligieron los materiales malacológicos de esta estructura porque sus etapas constructivas han contribuido al establecimiento de la cronología de Xochicalco -ya que en su mayoría tuvo un solo momento de edificación de arquitectura monumentaly por ello permiten hacer comparaciones temporales en cuanto a la adquisición y modificación de los moluscos.

Xochicalco se encuentra emplazado sobre cerros y

Recibido: 13 noviembre 2004; aceptado: 28 marzo 2007 barrancas en la parte norte del valle de Morelos, como los cerros Xochicalco, La Malinche y La Bodega en el estado de Morelos (Figs. 1B, 1C). A pesar de la vida efímera de este asentamiento, alrededor de 300 años, su posición estratégica dentro del valle y el control que ejerció sobre la ruta de circulación de productos (terrestres o marinos) de Michoacán y Guerrero, siguiendo la cuenca del río Balsas hacia el altiplano central (Kolb, 1987), permitió que este sitio concentrara y restringiera la distribución de determinados materiales en la región, como los exoesqueletos de moluscos marinos.

En este sentido, por un lado se tienen moluscos que no presentan modificaciones humanas o, a lo mucho, que presentan alteraciones producidas por la extracción del animal por percusión, palanqueo, hervido o abrasión (Waselkov, 1987), por lo cual sólo permiten la identificación taxonómica e inferir la zona y forma de obtención; otra función aparte de la alimenticia podría ser la de ofrenda votiva, producto almacenado y/o en preparación para la manufactura de objetos de concha, lo cual depende de la asociación contextual. Por otro lado, los objetos de concha, 


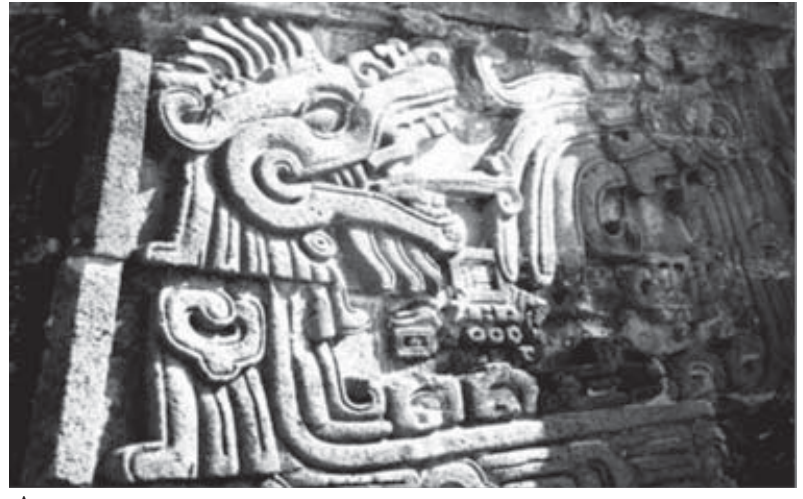

A

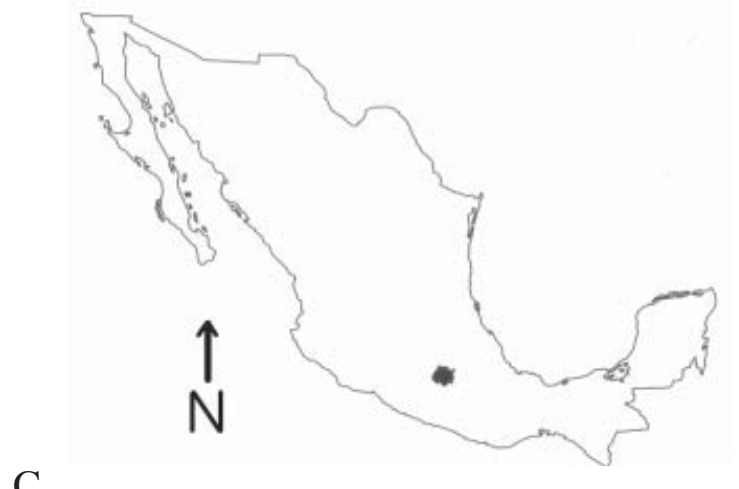

Figura 1. Localización de Xochicalco, Morelos. A, detalle de la fachada de la Pirámide de las Serpientes Emplumadas; B, vista aérea de Xochicalco; C, mapa de México indicando la ubicación de Xochicalco.

definidos como todas aquellas piezas manufacturadas por el hombre a partir de los exoesqueletos calcáreos de los moluscos (Velázquez, 1999), así como los residuos de su manufactura, permiten conocer qué herramientas, ornamentos y elementos votivos circulaban en distintas esferas sociales, su relación con el lugar de deposición y cuáles se elaboraban localmente, ya sea en talleres concentrados o en unidades dispersas (Hohmann, 2002).

\section{Material y métodos}

El material malacológico estudiado está conformado por 258 piezas, 242 completas y 16 fragmentos, que se encuentran depositadas en 4 lugares (en una bodega del "Proyecto Xochicalco" en el Centro INAH-Morelos de Cuernavaca, en el Museo de Sitio de Xochicalco, en el

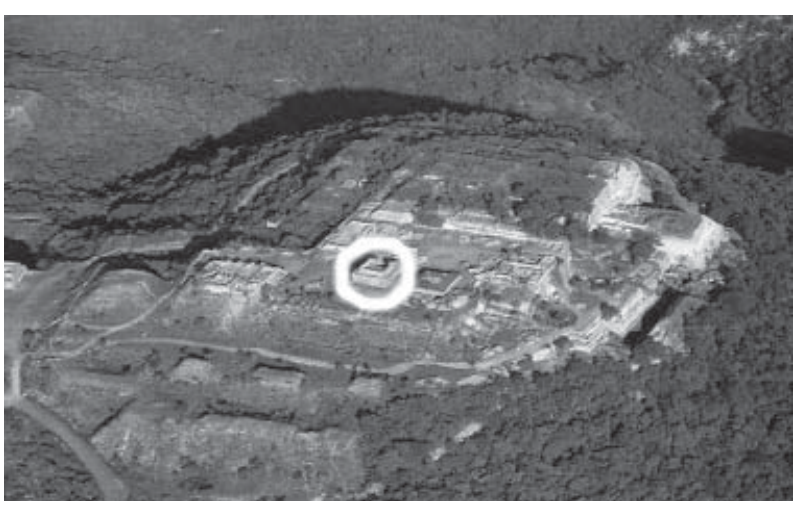

B

Museo Nacional de Antropología e Historia y en la bodega del Laboratorio de Arqueozoología del INAH, estos 2 últimos en la Ciudad de México). Proceden de las ofrendas descubiertas por Sáenz (1963) en 1962-1963 y el equipo de González Crespo et al. (en prensa) y Garza Tarazona en 1994, durante excavaciones realizadas en la Pirámide de las Serpientes Emplumadas en Xochicalco, Morelos. Las primeras corresponden a entierros secundarios de la etapa A (Fig. 2), un edificio anterior a la Pirámide de las Serpientes Emplumadas formado por un cuarto con altar y fechado para el 600 d.C. (González et al., en prensa). Estas ofrendas se caracterizan por presentar conjuntos de pendientes (Fig. 3A) o estar pigmentadas con cinabrio, sin importar que algunos de los moluscos sean de tonos rojizos, incrementando con ello su color. Dentro de ellas destaca la Ofrenda 1 compuesta por varias valvas de moluscos y sartales de pendientes de caracoles marinos, ubicada al centro de la estructura (Fig. 2), así como el Entierro 1 hallado entre los pilares, cuya ofrenda también presentó varios pendientes de caracoles marinos, un disco de piedra y 2 placas de jade decoradas con personajes antropomorfos (Sáenz, 1963). Además, las ofrendas recuperadas por el Proyecto Arqueológico Especial Xochicalco forman parte del ajuar funerario del Entierro 2, correspondiente a la etapa C de esta estructura (Fig. 2), cuando ya fue construida la Pirámide de las Serpientes Emplumadas hacia el 750-800 d.C. (González et al., en prensa). Se trata de un entierro primario descubierto al desmontarse la escalera de acceso, entre el tercero y quinto escalón, en una rotura del piso de la estructura. El esqueleto corresponde a un individuo entre los 13 y 17 años de edad, depositado en posición de decúbito lateral derecho flexionado, acompañado por una importante ofrenda funeraria constituida por un cráneo infantil, 5 cilindros tallados, 65 cuentas de piedra verde de distintos tamaños, 12 cuentas en forma de cantaritos, un sartal de 20 pendientes de caracol en forma de cráneos con incrustaciones de pirita en los ojos, un pectoral de concha, 


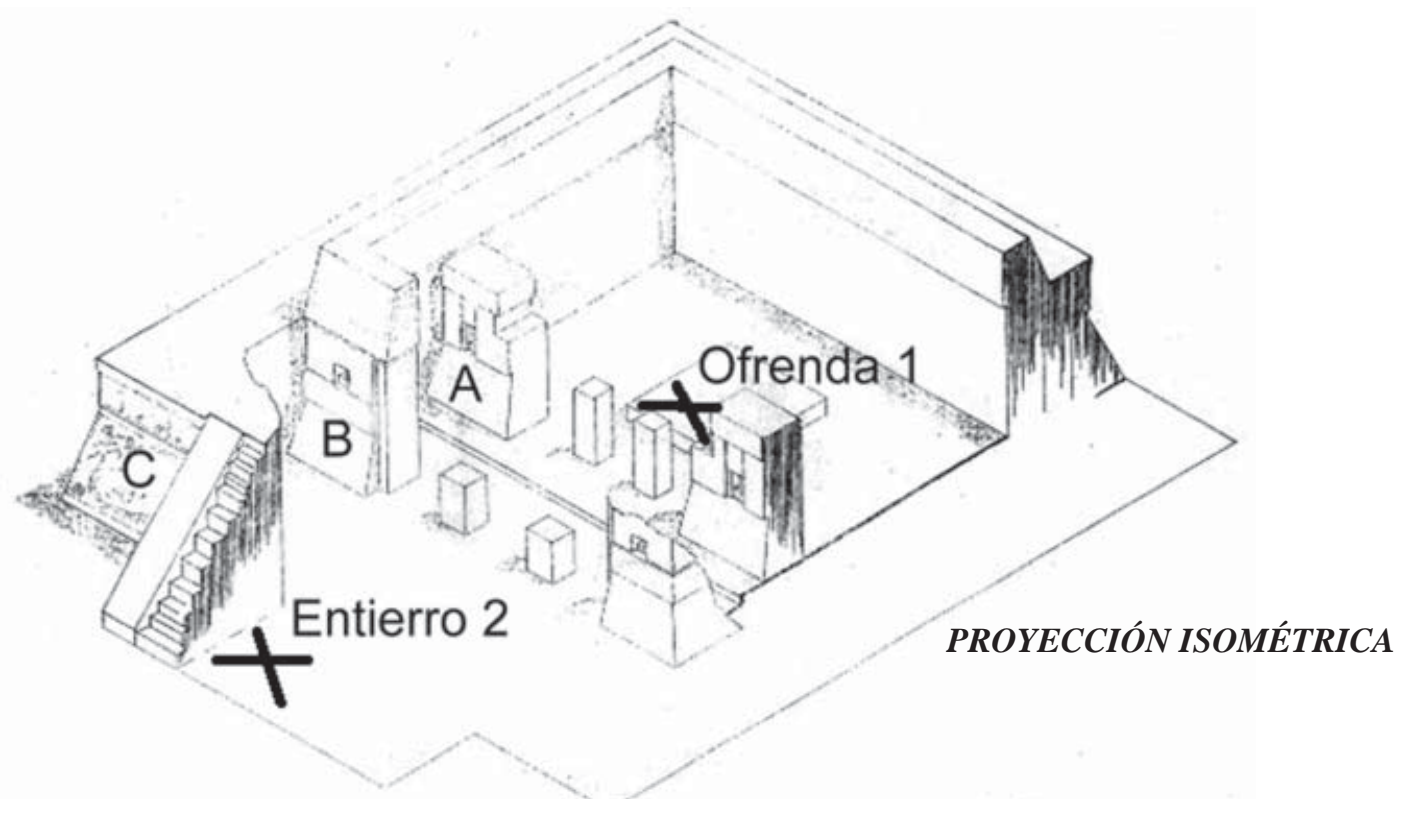

Figura 2. Localización de las ofrendas de la Pirámide de las Serpientes Emplumadas y sus 3 etapas arquitectónicas (A, B y C).

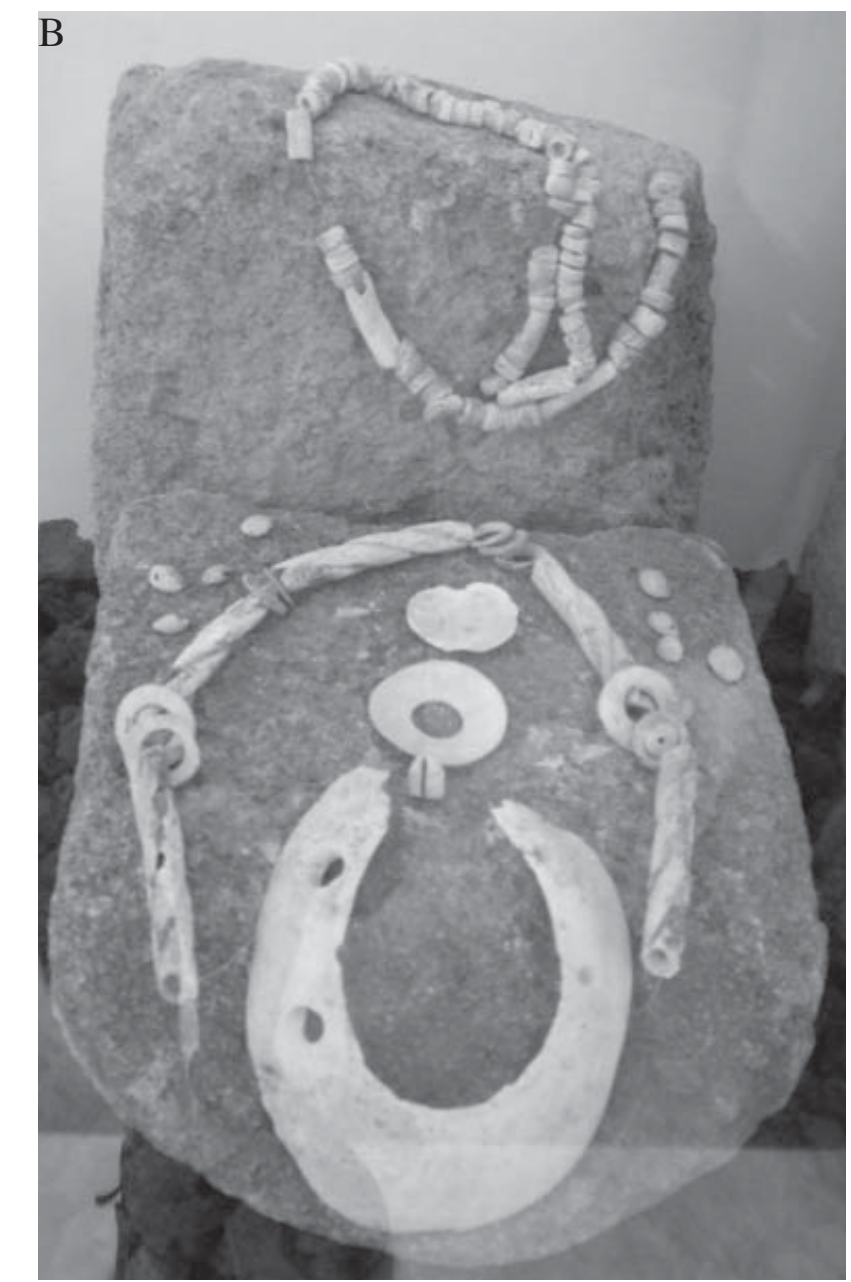

Figura 3. Moluscos ofrendados en la Pirámide de las Serpientes Emplumadas. A, pendientes automorfos en Oliva porphyria de la Ofrenda 1; B, objetos en Strombus gigas, Spondylus princeps, Patella mexicana, Trivia radians y Jenneria pustulata del Entierro 2. 
varias cuentas nacaradas, varios pendientes de caracol (Fig. 3B), cuentas de barro, algunos fragmentos de un vaso de alabastro, espinas de maguey y carbón (Garza, 1995).

Para la identificación de las especies se utilizaron las obras de Keen (1971) para el Pacífico, Morris (1973) para el Caribe y Vokes y Vokes (1983) para la Península de Yucatán. También se consultaron las colecciones de referencia de moluscos de la Subdirección de Laboratorios y Apoyo Académico del INAH en la Ciudad de México. Cabe señalar que parte de la colección en estudio, aquélla depositada en el Laboratorio de Arqueozoología M. en C. Ticul Álvarez, ya había sido identificada por el biólogo Eduardo Corona. Por lo cual se han incluido en este análisis las especies señaladas por ese investigador.

Después se realizó un análisis tipológico para clasificar todas y cada una de las piezas modificadas por el hombre que integran esta colección, basado en aspectos morfológicos y funcionales. Para ello se siguió el esquema propuesto por Suárez Díez (1977) en su clásica obra Tipología de los objetos prehispánicos de concha, pero con las modificaciones hechas por Velázquez Castro (1999) en su libro Tipología de los objetos de concha del Templo Mayor de Tenochtitlan.

Posteriormente se efectuó un análisis de las huellas de manufactura de los objetos de concha, basado en los estudios realizados en el "Proyecto de arqueología experimental en materiales conquiológicos" y el "Proyecto de técnicas de manufactura del México prehispánico", ambos bajo la dirección de Velázquez (2004). En el primero, han sido reproducidos en el taller de arqueología experimental los diferentes tipos de modificaciones que presentan los objetos -percusiones, desgastes, cortes, perforaciones, incisiones, calados y acabados-, partiendo de la obra de Suárez (1974). Para ello se han empleado las herramientas y procesos que, por diversas fuentes de información, es posible suponer que fueron utilizados por los distintos pueblos del México prehispánico, como aparece en el Cuadro 1.

Con base en ello, las huellas de manufactura resultantes fueron comparadas tanto macroscópica como microscópicamente con el material arqueológico. El empleo del microscopio electrónico de barrido (MEB) modelo JEOL JSM-6460LV en el segundo proyecto ha ofrecido los mejores resultados para poder distinguir las características morfológicas de la superficie de los materiales: topología, rugosidad, porosidad y tamaño de las partículas que los constituyen. A su vez, para llevar a cabo las comparaciones entre las diferentes muestras, las micrografías fueron obtenidas empleando los mismos parámetros (modo $\mathrm{HV}$, haz de 45 , voltaje de $20 \mathrm{kv}$ y distancia de 10mm) y observadas en 4 amplificaciones: 100x, 300x, 600x y 1000x (Velázquez, 2004). Asimismo, para agilizar el análisis, en dicho proyecto se realizan réplicas en polímeros, lo cual permite ingresar varias modificaciones a la vez (hasta 20). Con ello han podido obtenerse huellas de piezas que superan el tamaño de la cámara de muestreo, mayores a $10 \mathrm{~cm}$, o de algunas modificaciones de difícil observación, como las paredes internas de las perforaciones. Esta técnica ha permitido incursionar en el estudio de la eficacia de las herramientas y los tiempos invertidos en la elaboración de objetos, así como en la identificación de preferencias culturales y tradiciones tecnológicas en diferentes regiones de México (Velázquez et al., 2004; Velázquez y Melgar, 2003).

Cuadro 1. Tipos de modificaciones y herramientas empleadas

\begin{tabular}{|c|c|}
\hline Modificación & Herramientas \\
\hline Desgastes de superficies & $\begin{array}{c}\text { Basalto, andesita, riolita, arenisca, caliza y granito, adicionando agua y } \\
\text { ocasionalmente arena. }\end{array}$ \\
\hline Cortes & $\begin{array}{l}\text { Arena, agua y tiras de piel o cuerdas vegetales. } \\
\text { Herramientas líticas de pedernal y obsidiana }\end{array}$ \\
\hline Perforaciones & $\begin{array}{c}\text { Abrasivos (arena, ceniza volcánica, polvo de obsidiana, polvo de pedernal y polvo } \\
\text { de cuarzo), animados con ramas de carrizo, adicionando agua. } \\
\text { Herramientas líticas de pedernal y obsidiana. }\end{array}$ \\
\hline Calados & $\begin{array}{l}\text { Abrasivos (arena, ceniza volcánica, polvo de obsidiana, polvo de pedernal y polvo } \\
\text { de cuarzo), animados con ramas de carrizo de gran diámetro, adicionando agua. }\end{array}$ \\
\hline Incisiones & Herramientas líticas de pedernal y obsidiana. \\
\hline Acabados & $\begin{array}{l}\text { Pulido con abrasivos, agua y trozos de piel. } \\
\text { Bruñidos con trozos de piel en seco. } \\
\text { La aplicación de ambos acabados }\end{array}$ \\
\hline
\end{tabular}




\section{Resultados}

Los moluscos identificados abarcan 12 especies, 9 de la provincia Panámica-Pacífica y 3 de la del Caribe. Del Pacífico se obtuvieron 196 piezas: Jenneria pustulata, 2; Muricanthus princeps, 3; Oliva incrassata, 2; O. julieta, 1; O. porphyria, 52; Patella mexicana, 1; Pinctada mazatlanica, 34; Spondylus princeps, 92 y Trivia radians, 1. Del Atlántico se tiene a Marginella cf. apicina con 13 piezas; Pleuroploca gigantea con 15 y Strombus gigas con 33 .

Respecto a la tipología de los objetos, en las ofrendas a entierros secundarios se observaron 66 piezas de especies que provienen exclusivamente del Pacífico; 13 de ellas no presentan modificación alguna. En estas circunstancias están 2 ejemplares de $O$. porphyria, 8 de $S$. princeps y 3 de $M$. princeps. Los únicos objetos elaborados son los pendientes automorfos (aquellos que no pierden la forma natural del caracol con el cual están manufacturados), todos del género Oliva: 50 de O. porphyria, 2 de O. incrassata y 1 de O. julieta (Fig. 3A).

La ofrenda del entierro primario presenta una mayor diversidad de especies y objetos. Se contaron 191 objetos, 130 hechos con 5 especies del Pacífico y 61 con 3 especies de las costas del Golfo de México. Así, los tipos de piezas por especie quedaron clasificados de la siguiente manera: 1 pectoral de $P$. mexicana, 13 pendientes de $M$. cf. apicina, 2 de $J$. pustulata y 1 diente de $T$. radians, 33 cuentas y una incrustación de $P$. mazatlanica, 84 cuentas de $S$. princeps, 7 incrustaciones, 7 cuentas y un pectoral de $P$. gigantea, y 8 cuentas, 8 incrustaciones y 17 pendientes de $S$. gigas (Fig. 3B).

En cuanto al análisis de las huellas de manufactura con microscopía electrónica de barrido, las 44 piezas elegidas dieron los siguientes resultados: a) 16 piezas presentaron la cara dorsal o ventral cruzada por bandas irregulares y redondeadas, del orden de las $100 \mu \mathrm{m}$ de espesor, las cuales coincidieron con las producidas por el desgaste experimental con basalto (Figs. 4A, 4B), mostrando una notable diferencia con las superficies trabajadas con otras lajas, como riolita o arenisca (Figs. 4C, 4D): 7 en S. gigas, 3 en P. mazatlanica, 2 en P. gigantea, 2 en $S$. princeps, 1 en $P$. mexicana y 1 en $T$. radians; $b) 14$ piezas mostraron bordes atravesados por líneas finas rectasparalelas o sinuosas de $2 \mu \mathrm{m}$ de anchura, similares a las huellas producidas por el corte experimental con lascas de obsidiana: 7 en S. gigas, 3 en P. mazatlanica, 2 en $S$. princeps y 2 en $P$. gigantea; c) 7 piezas con horadaciones cruzadas por bandas rectas-paralelas de aproximadamente $5 \mu \mathrm{m}$ de espesor, llegando a formar rebordes de mayores dimensiones y rupturas disparejas de la estructura cristalina de la concha, similares a las huellas de perforaciones experimentales hechas con lascas de pedernal (Figs. 5A, 5B): 4 en S. gigas, 2 en S. princeps y 1 en P. mazatlanica; d) 4 piezas presentaron paredes atravesadas por líneas de aproximadamente $3 \mu \mathrm{m}$ de anchura, las cuales se unen para formar rebordes de mayores dimensiones, similares a las perforaciones y calados producidos experimentalmente empleando arena y carrizo (Figs. 5C, 5D): 4 piezas, todas con Oliva.e) 2 piezas presentaron líneas finas de 3 $\mu \mathrm{m}$ de anchura, similares a las incisiones experimentales realizadas con lascas de obsidiana: 1 cuenta helicoidal y 1 pendiente en forma de cráneo hechos con S. gigas; $f$ ) 4 piezas presentaron en los calados líneas finas de $2 \mu \mathrm{m}$, similares a las hechas con lascas de obsidiana, algunas de las cuales estaban regularizadas con pequeñas lajas de basalto, debido a las bandas de $100 \mu \mathrm{m}$ de espesor sobre los rasgos anteriores (Figs. 6A, 6B) y siendo muy diferentes a las producidas mediante el empleo de lascas de pedernal o ceniza volcánica (Figs. 6C, 6D): 4 pendientes, todos con especies del género Oliva.

\section{Discusión}

Con la información anterior podemos señalar algunos aspectos importantes a tomar en consideración para futuras investigaciones en Xochicalco y, por extensión, en otros sitios prehispánicos con materiales malacológicos. En primer lugar, en este estudio se resalta la necesidad de llegar en la identificación taxonómica al nivel de especie, ya que las costas de ambos océanos, Pacífico y Atlántico, comparten géneros, como Oliva, Spondylus, Strombus y Pinctada, y sin lo cual no podrían rastrearse las zonas de obtención de materiales, las provincias malacológicas de procedencia ni inferirse antiguas rutas de intercambio. También con ello se podría reevaluar el abastecimiento de moluscos que se ha aplicado en contextos mesoamericanos, ya que comúnmente se cree que los moluscos hallados en asentamientos del interior proceden de las playas más cercanas, obviándose en muchos casos largas rutas de intercambio de épocas tan antiguas como el Preclásico tardío (Pires-Ferreira, 1978; Barrera Vázquez, 1980). Esto sale a colación debido a que no todos los moluscos arqueológicos hallados en Xochicalco corresponden al océano Pacífico, su costa más cercana; por el contrario, puede apreciarse que también hay especies del Atlántico presentes en la colección, las cuales seguramente llegaron de las costas del Golfo de México.

De la provincia malacológica panámica-pacífica, que va desde el mar de Cortés hasta Tumbes, Perú (Keen, 1971), se obtuvieron 143 piezas de moluscos, mientras que la del Caribe, que incluye casi la totalidad del Golfo de México, Florida y el mar Caribe hasta el norte de Brasil 


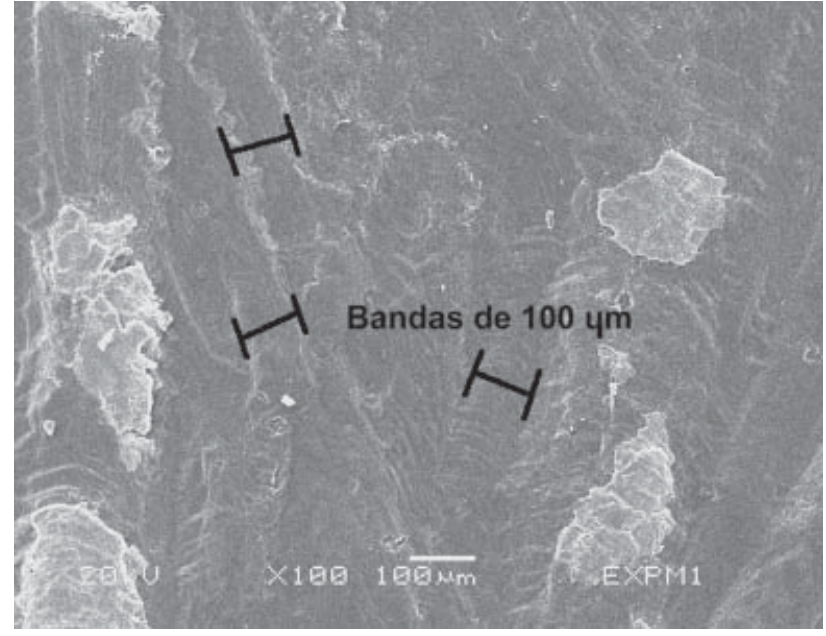

A

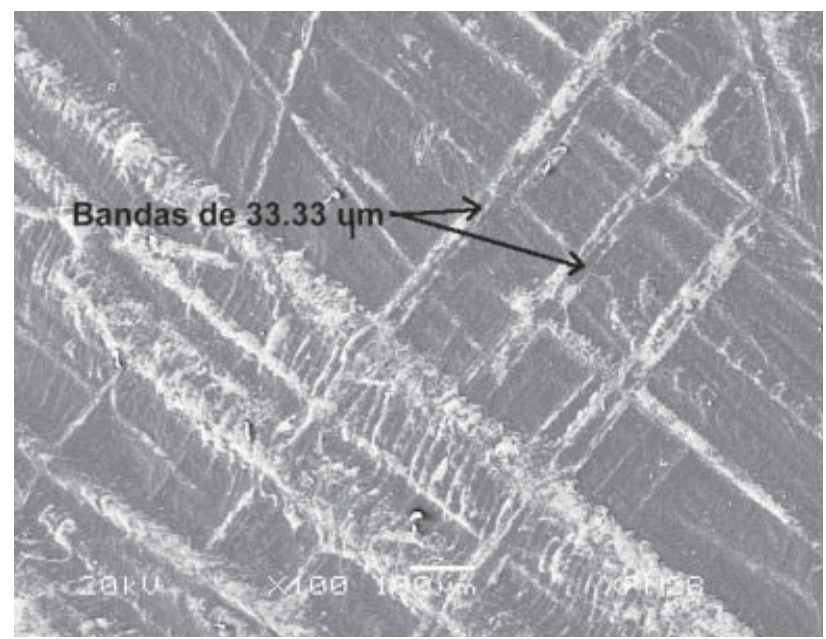

$\mathrm{C}$

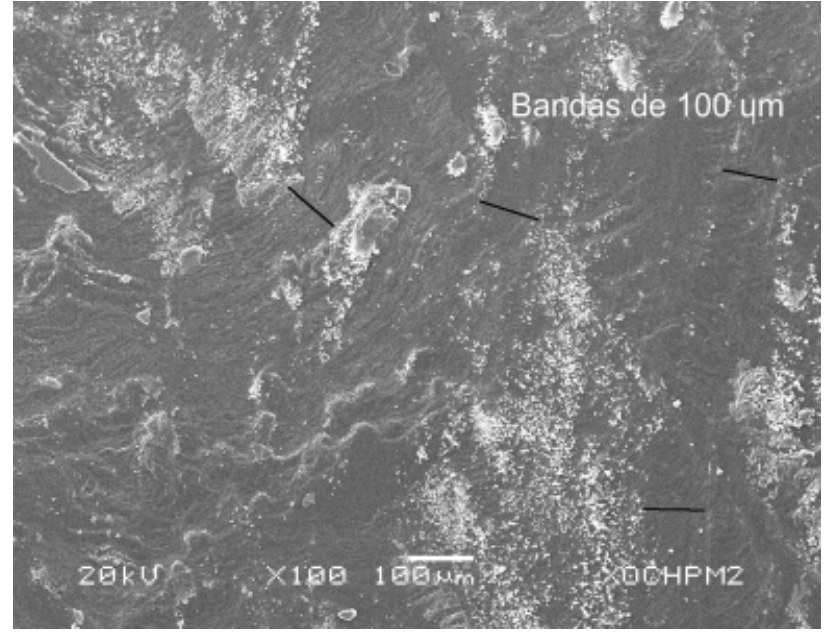

B

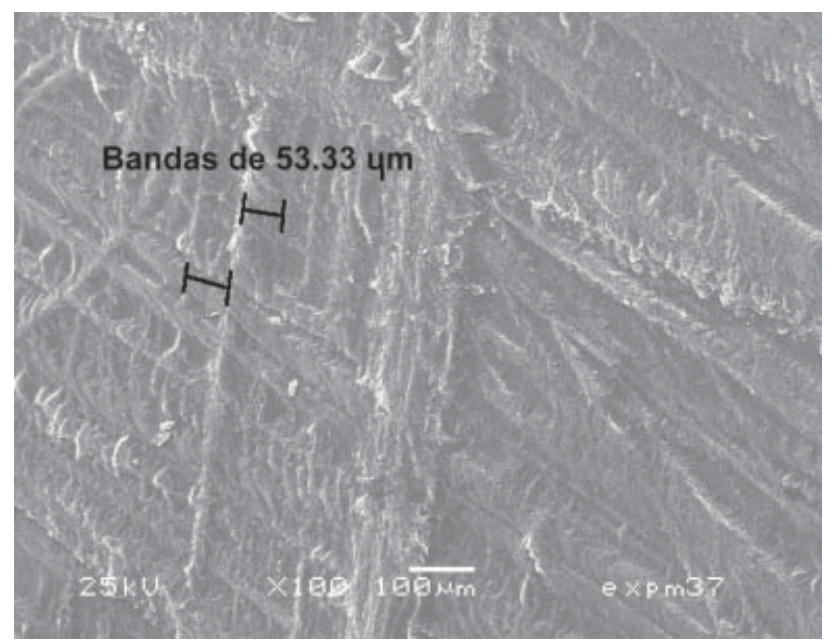

$\mathrm{D}$

Figura 4. Huellas de desgastes analizados con icroscopía electrónica de barrido. A, con basalto experimental a 100x; B, superficie arqueológica en Pinctada mazatlanica a 100x; C, con riolita experimental a 100x; D, con arenisca experimental a 100x.

(Morris, 1973), se obtuvieron sólo 48. Se ha dicho que esta mayor proporción de especies provenientes de las costas del Pacífico puede deberse a que Xochicalco tuvo afiliaciones culturales muy fuertes con algunas porciones del noreste de Guerrero, la depresión del Balsas y el valle de Toluca (Hirth y Cyphers, 1988), que posiblemente fueron la causa de esta diferencia en la mayor adquisición de recursos y productos de las costas de Michoacán y Guerrero hacia el altiplano central y vinculándose con grupos del occidente de México. No en vano el $85 \%$ de la obsidiana de Xochicalco es de tonos grisáceos y procede de Zinapécuaro, Michoacán (Sorensen et al., 1989).

Son notables las diferencias existentes entre las piezas de concha depositadas en las ofrendas de acuerdo con su temporalidad. Por un lado está la poca diversidad de los moluscos de los entierros secundarios, ya que todos son exclusivamente de las costas del Pacífico; además, la mayoría están sin modificar, aunque hay algunos pigmentados con cinabrio, así como los sartales de un solo tipo de objeto, es decir, los pendientes automorfos de O. porphyria, O. julieta y O. incrassata. Aunado a lo anterior, cabe señalar que son los más antiguos de esa estructura, correspondientes a la etapa A, fechada para el 600 d.C. (González et al., en prensa). Por el otro lado, los moluscos del ajuar del entierro primario, fechado para la etapa C de esta estructura, ya construida la Pirámide de las Serpientes Emplumadas, hacia 750-800 d.C. (González et al., en prensa), presentan la mayor variedad de especies 


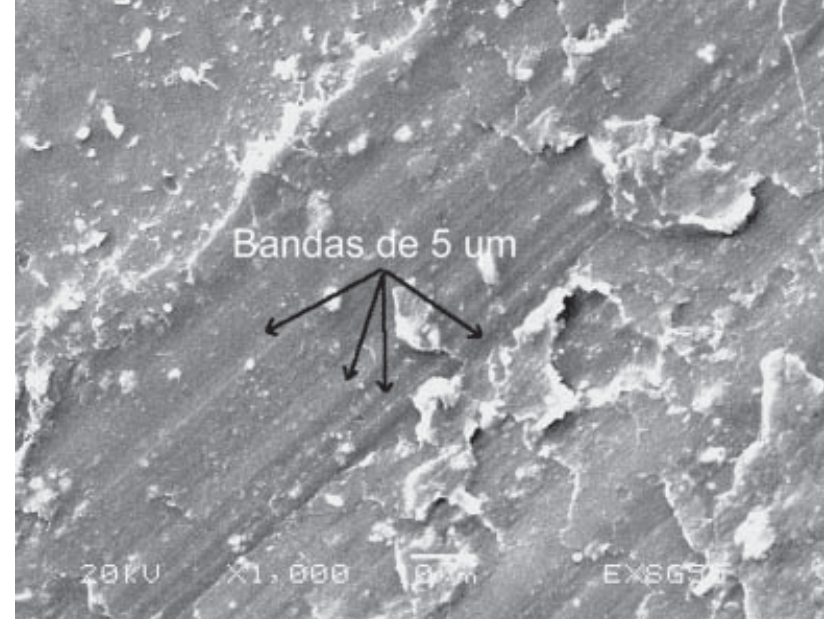

A

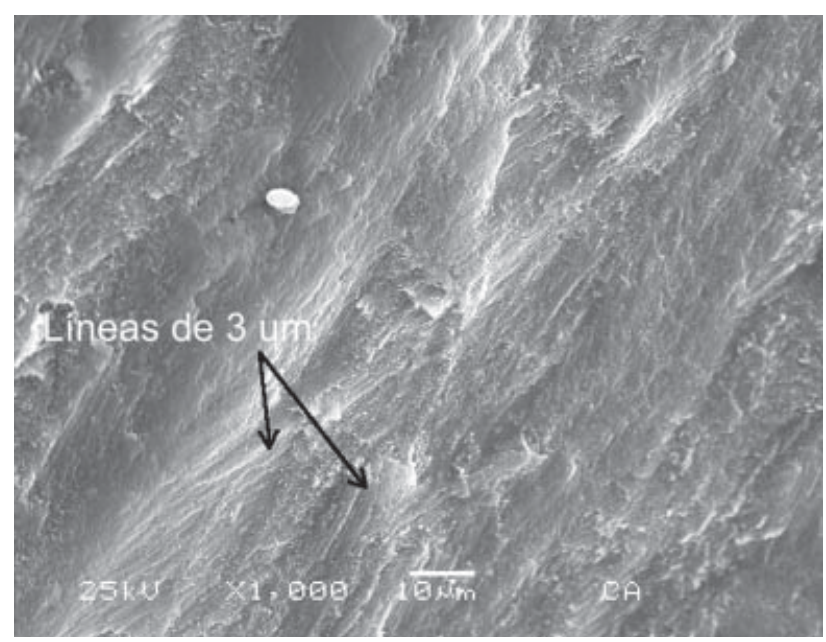

$\mathrm{C}$

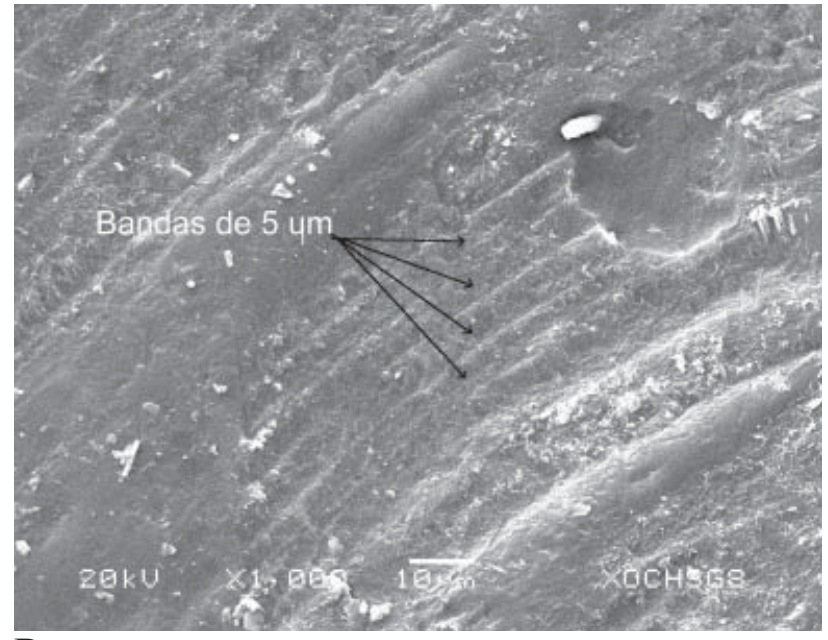

B

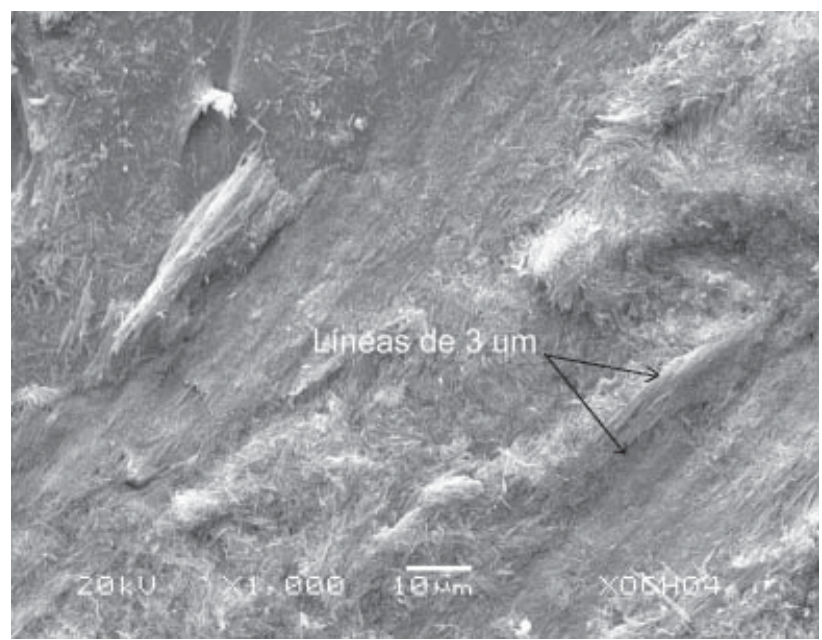

$\mathrm{D}$

Figura 5. Huellas de perforaciones analizadas con Microscopía Electrónica de Barrido. A, con lascas de pedernal experimentales a 1000x; B, horadación en pendiente arqueológico de Strombus gigas a 1000x; C, con carrizo y arena experimental a 1000x; D, horadación en pendiente arqueológico de Oliva porphyria a 1000x.

y objetos, incluyendo 3 especies caribeñas, M. cf. apicina, S. gigas y P. gigantea, lo que podría indicar una apertura de rutas con el Golfo de México, o el establecimiento de nexos con grupos de aquella región. También, el tiempo invertido en la elaboración de estos objetos (cuentas, pendientes xenomorfos -aquellos que pierden la forma natural del molusco del cual están hechos- y pectorales) es mayor en comparación con el invertido en elaborar los pendientes automorfos de las primeras ofrendas, ya que la mayoría presenta más de una modificación en su manufactura.

Enlazado a lo anterior y con base en los resultados obtenidos por el análisis con el MEB, cabe destacar la estrecha gama de herramientas y modificaciones en los objetos de las primeras ofrendas, donde solamente se identificaron lascas de obsidiana, arena y carrizo para perforaciones y calados; frente a los objetos de las ofrendas más tardías, donde se utilizaron lajas de basalto para desgastes, lascas de obsidiana para los cortes y lascas de pedernal para las perforaciones, sin importar la especie trabajada. Ello obliga a revisar cuáles herramientas corresponden con las materias primas locales y cuáles pudieron traerlas de fuera. Así, en relación con los materiales disponibles en la región, hay calizas, lutitas, areniscas, tobas basálticas y andesíticas, así como hojas de pedernal de la Formación Xochicalco (Hirth y Cyphers, 


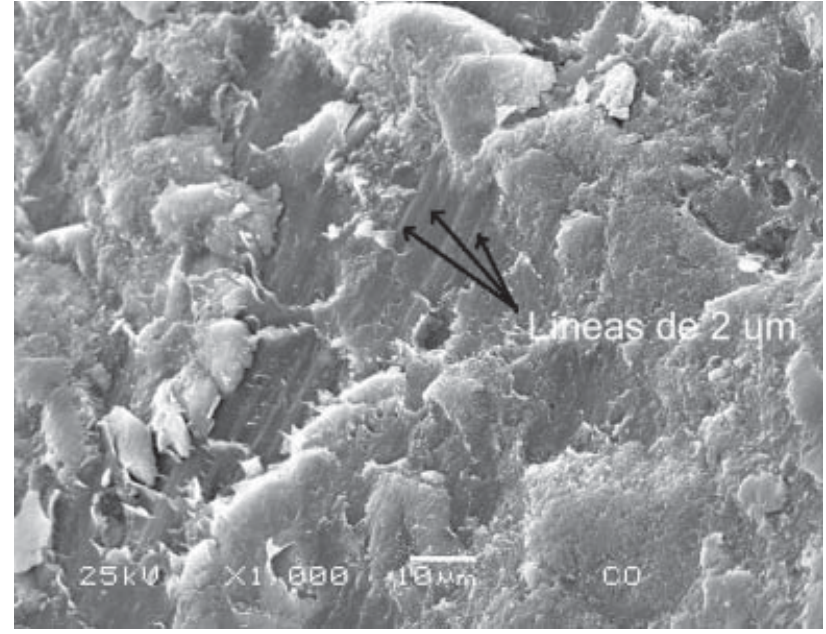

A

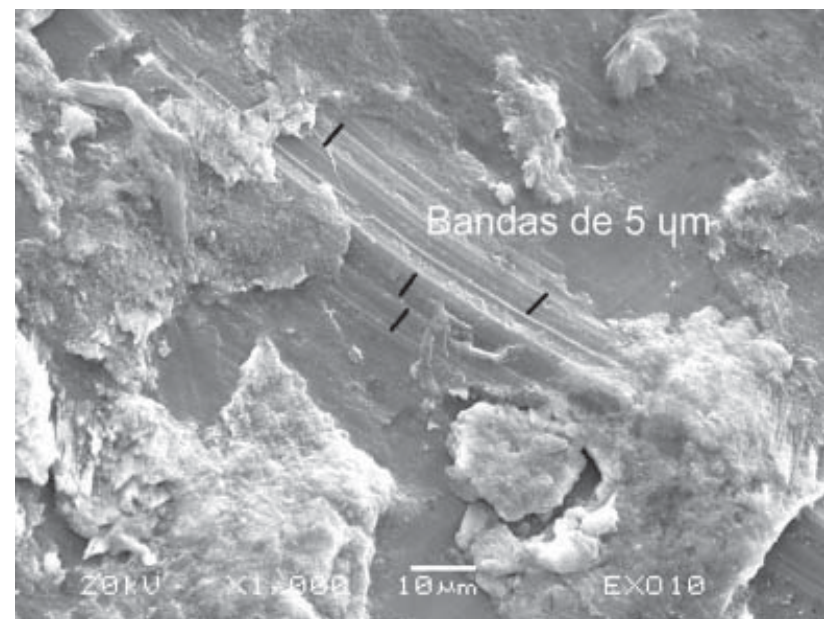

$\mathrm{C}$

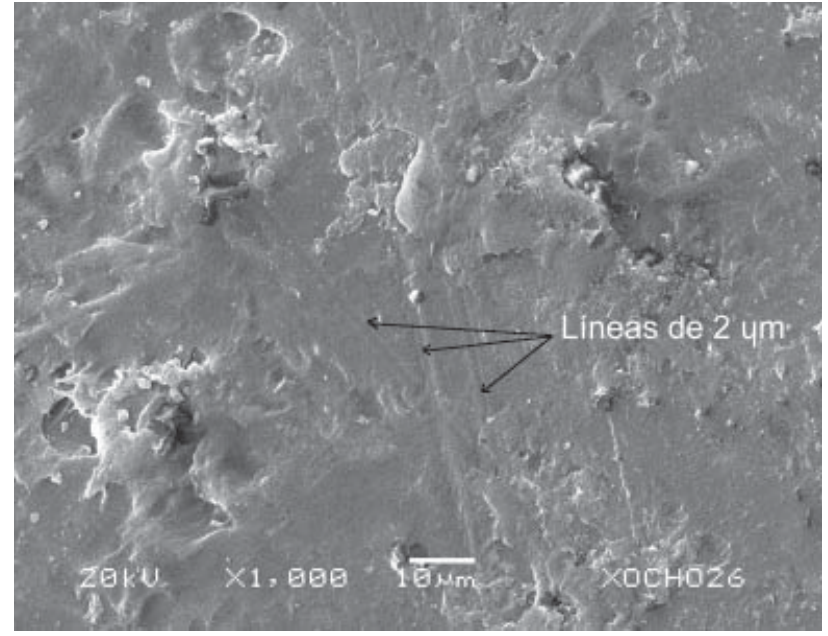

B

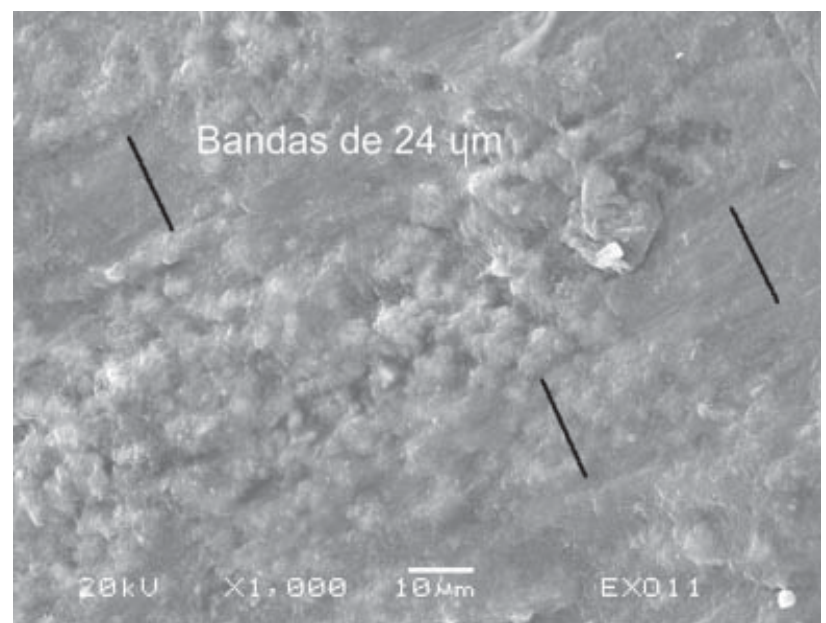

$\mathrm{D}$

Figura 6. Huellas de calados analizados con microscopía electrónica de barrido. A; calado experimental con lascas de obsidiana a 1000x; B, pared del calado arqueológico en pendiente automorfo de Oliva porphyria a 1000x; C, calado experimental con lascas de pedernal a 1000x; D, calado experimental con ceniza volcánica y carrizo a 1000x.

1988), cuya compactación y dureza son de mala calidad para funcionar como herramientas de trabajo, pues se exfolian y astillan fácilmente. Estas deficiencias en el pedernal local se reflejan en la escasez de herramientas hechas con este material (Sorensen et al., 1989), lo cual permite pensar que el pedernal utilizado en la manufactura de estos objetos pudo ser adquirido en Oaxtepec, donde hay un yacimiento de buena calidad que incluso fue explotado por los mexicas durante el Posclásico (Sahagún, 1956). Mientras que el basalto empleado en el sitio, principalmente en metates o con fines arquitectónicos, se ha dicho que procede del grupo Chichinautzin al este de Xochicalco, donde abunda el basalto negro (Hirth y Cyphers, 1988). En cuanto a la obsidiana, proviene sobre todo de los yacimientos de
Zinapécuaro en Michoacán (Sorensen et al., 1989), como se señaló anteriormente.

Por último, se aprecian diferencias en el control de la producción de los objetos de ambos tipos de ofrendas. Así, en las más tempranas la variedad de especies y de objetos es menor, exclusivamente del Pacífico, sólo hay pendientes automorfos elaborados con el género Oliva y restringidos sólo a este momento, donde hay varias calidades en cuanto al tipo de perforación o calado circular, pues en algunos las paredes no se regularizaron, llamando la atención que ello no incidiera en la conformación de los sartales. En contraste, es notable la diversidad de las más tardías, que incluyen 3 especies del Caribe, M. cf. apicina, S. gigas y P. gigantea, así como piezas con más modificaciones, 
debidas al mayor tiempo de trabajo invertido en su elaboración, y cuya similitud en la forma y homogeneidad en las huellas de manufactura sugieren un mayor control en su producción, quizás en áreas concentradas o talleres bajo supervisión de la élite, y que éstos se encontraran en el sitio mismo. Este último patrón se ha dicho que responde a las necesidades de los grupos gobernantes por controlar la elaboración y distribución de objetos suntuarios o bienes de prestigio, los cuales generalmente son hechos de materiales exóticos y por ende escasos, y de acceso restringido; por ello son usados como símbolos de poder ideológicos, políticos o religiosos, al mismo tiempo que permiten a la élite mantener su jerarquía y diferenciación social con el resto de la sociedad (Brumfiel y Earle, 1987; Hohmann, 2002).

\section{Agradecimientos}

Se agradece a los integrantes del Proyecto Arqueológico Especial Xochicalco, Norberto González, Silvia Garza, Claudia Alvarado y Beatriz Palavicini, por las facilidades otorgadas durante la realización de este estudio. También a los miembros del taller de arqueología experimental en concha y su responsable, Adrián Velázquez, por la asesoría en las huellas de manufactura y los experimentos realizados. Asimismo, a Belem Zúñiga y Norma Valentín, por la asesoría en la identificación taxonómica de los materiales. Y a José Antonio Alva por la operación del MEB para obtener las micrografías.

\section{Literatura citada}

Barrera Vásquez, A. 1980. Diccionario maya Cordemex. Maya-español / español-maya. Cordemex, Mérida, Yucatán.

Barrera Rubio, A. 1985. Litoral-marine economy at Tulum, Quintana Roo, México. In The lowland Maya Postclassic, A. F. Chase y P. M. Rice (eds.). University of Texas Press, Austin. p. 50-61.

Brumfiel, E. M. y T. K. Earle. 1987.

Specialization, exchange, and complex societies: an introduction. In Specialization, exchange, and complex societies, E. M. Brumfiel y T. K. Earle (eds.) Cambridge University Press, Cambridge. p. 1-9.

Garza, I. 1995. Proyecto Xochicalco (1993-1994). Informe del material óseo. Archivo del Centro INAH-Morelos, Cuernavaca. $14 \mathrm{p}$.

González, N., S. Garza, B. Palavicini y C. Alvarado. En prensa. Xochicalco chronology revisited. American antiquity. Society for American Archaeology, Washington, D.C. 25 p.
Hirth, K. G. y A. Cyphers. 1988. Tiempo y asentamiento en Xochicalco. Instituto de Investigaciones Antropológicas-Universidad Nacional Autónoma de México, México, D.F.

Hohmann, B. M. 2002. Preclassic Maya shell ornament production in the Belize Valley, Belize. Tesis doctorado, The University of New Mexico. Albuquerque. 254 p.

Keen, M. A. 1971. Sea shells of tropical west America. Stanford University Press, California. 1064 p.

Kolb, C. C. 1987. Marine shell trade and Classic Teotihuacan. BAR International Series 364, México, D.F. 227 p.

Morris, P. A. 1973. A field guide to shells of the Atlantic. The Peterson Field Guide Series, Houghton Mifflin , Boston, Massachusetts. 330 p.

Pires-Ferreira, J. W. 1978. Shell and iron-ore mirror exchange in Formative Mesoamerica, with comments on other commodities. In The ancient Mesoamerica village, K. Flannery (ed.). Academic, New York. p. 311-328.

Sáenz, C. A. 1963. Exploraciones en la Pirámide de las Serpientes Emplumadas, Xochicalco. Revista Mexicana de Estudios Antropológicos 19:7-25.

Sahagún, B. 1956. Historia general de las cosas de la Nueva España, 4 vols. Porrúa, México, D.F. 1093 p.

Sorensen, J. H., K. G. Hirth y S. M. Ferguson. 1989. The contents of seven obsidian workshops around Xochicalco, Morelos. In La obsidiana en Mesoamérica, Colección Científica 176, M. Gaxiola y J. E. Clark (coords.). Instituto Nacional de Antropología e Historia, México, D.F. p. 269-275.

Suárez, L. 1974. Técnicas prehispánicas en los objetos de concha. Instituto Nacional de Antropología e Historia, México, D.F. 130 p.

Suárez Díez, L. 1977. Tipología de los objetos prehispánicos de concha. Instituto Nacional de Antropología e Historia, México, D.F. 210 p.

Suárez, L. 2002. Tipología de los objetos prehispánicos de concha, segunda edición, Porrúa e Instituto Nacional de Antropología e Historia, México, D.F. 240 p.

Velázquez Castro, A. 1999. Tipología de los objetos de concha del Templo Mayor de Tenochtitlan. Colección Científica 392, Instituto Nacional de Antropología e Historia, México, D.F. 134 p.

Velázquez, A. 2004. Técnicas de manufactura de los objetos de concha del Templo Mayor de Tenochtitlan. Tesis doctorado Instituto de Investigaciones Antropológicas, Facultad de Filosofía y Letras, Universidad Nacional Autónoma de México. México, D.F. 240 p.

Velázquez, A. y E. Melgar. 2003. La elaboración de los ehecacózcatl de concha del Templo Mayor de Tenochtitlan. In Homenaje a Eduardo Matos 
Moctezuma, Leonardo López Luján (coord.) Instituto Nacional de Antropología e Historia, México, D.F. 18 p.

Velázquez, A., D. Mendoza y N. Valentín. 2004. Los anahuatl de concha del Templo Mayor de Tenochtitlan. Su valor visto a través de sus técnicas de manufactura. In La ciencia de materiales y su impacto en la arqueología, D. Mendoza, E. L. Brito y J. A. Arenas (eds.) Academia Mexicana de Ciencia de Materiales,
México, D.F. p. 129-140.

Vokes, A. y E. Vokes. 1983. Distribution of shallow water marine Mollusca, Yucatan Peninsula, Mexico. Monograph 1. Mesoamerican Ecological Institute, Tulane University, New Orleans. 183 p.

Waselkov, G. 1987. Shellfish gathering and shell midden archaeology. In Advances in archaeological method and theory 10, M. B. Schiffer (ed.). Academic, Tucson, Arizona. p. 93-210. 